\title{
Design of Wide-Band Bandpass Filter Using Composite Right/Left-Handed Transmission Line Structure
}

\author{
Baoping Ren, ${ }^{1,2}$ Haiwen Liu, ${ }^{1}$ Xuehui Guan, ${ }^{1}$ Pin Wen, ${ }^{1}$ Xiang Xiao, ${ }^{1}$ and Zhewang Ma $^{2}$ \\ ${ }^{1}$ School of Information Engineering, East China Jiaotong University, Nanchang 330013, China \\ ${ }^{2}$ Graduate School of Science and Engineering, Saitama University, Saitama 338-8570, Japan \\ Correspondence should be addressed to Baoping Ren; rbp2008@126.com
}

Received 27 November 2015; Revised 19 March 2016; Accepted 29 March 2016

Academic Editor: Jiun-Wei Horng

Copyright (c) 2016 Baoping Ren et al. This is an open access article distributed under the Creative Commons Attribution License, which permits unrestricted use, distribution, and reproduction in any medium, provided the original work is properly cited.

\begin{abstract}
A wide-band microstrip bandpass filter (BPF) based on the improved composite right/left-handed transmission line (CRLH-TL) structure is presented in this paper. Compared to the traditional CRLH-TL with via hole, the improved one is an all-planar structure, which owns the advantage of fabrication and loss. The equivalent lossless LC circuit model of the proposed structure is established. EM software Sonnet is adopted to design the wide-band filter with bandwidth of $1.4 \mathrm{GHz}$ (from $1.9 \mathrm{GHz}$ to $3.3 \mathrm{GHz}$ ). The circuit occupies only $20.6 \times 12.8 \mathrm{~mm}^{2}$. Finally, the fabrication and measurement are implemented. A good agreement between simulation and measured results verifies the validity of the design methodology.
\end{abstract}

\section{Introduction}

In recent years, left-handed materials (LHMs) have received substantial attention in the scientific and engineering communities for their specific electromagnetic (EM) property (simultaneously negative permittivity $\varepsilon$ and permeability $\mu$ ) [1]. Science magazine named LHMs as one of the top ten scientific breakthroughs of 2003 [2]. The first artificial LHM composed of thin copper wires and copper split-ring resonators (SRRs) was realized by Smith group at the University of California [3]. However, this kind of structure is always difficult to implement for microwave applications because resonant units such as SRRs are lossy and narrow-banded [4]. Several researchers soon realized that transmission lines (TLs) approach towards LHMs was possible $[5,6]$. In actual implementation, LHMs are considered to be a more general model of composite right/left-handed (CRLH) TLs, which also include right-handed (RH) effects that occur naturally in LH TLs. With CRLH structure, many new kinds of microwave devices are designed for low loss and broadband characteristics, such as divider, phase shifter, coupler, and leaky-wave antenna [7].

New ultra-wide-band radar and high-rate communication systems require very specialized RF devices capable of operating over wide frequency scope [8]. Thus, as the key element in RF system, high efficient wide-band bandpass filter (BPF) is no exception in this background. The popular way of designing wide-band filters is employing multiplemode resonators $[9,10]$ or signal-interference concepts $[11$, 12]. However, the circuit sizes of the aforementioned are comparatively large. More recently, CRLH TL unit cells are applied to design compact wide-band filter $[13,14]$. Nevertheless, a via hole or dual-layer structure is indispensable, which complexes the fabrication process. In our previous works, an all-planar CRLH resonator is proposed and fabricated on high-temperature superconducting film [15], which obtains a high performance filtering response with ultralow insertion loss, but the bandwidth is small.

In this paper, an improved CRLH TL is proposed to design a wide-band BPF. Patch capacitance replaces traditional via hole connected to shunt inductance in this new structure cell, which reduces the fabrication difficulty and circuit loss. The filter is simulated and optimized by EM software Sonnet and the obtained circuit size is $0.3 \lambda_{g} \times$ $0.18 \lambda_{g}\left(\lambda_{g}\right.$ is the guided wavelength of the $50 \Omega$ line at the center frequency). To observe the LH property, the dispersion diagram is given and researched in the paper. Simulation 


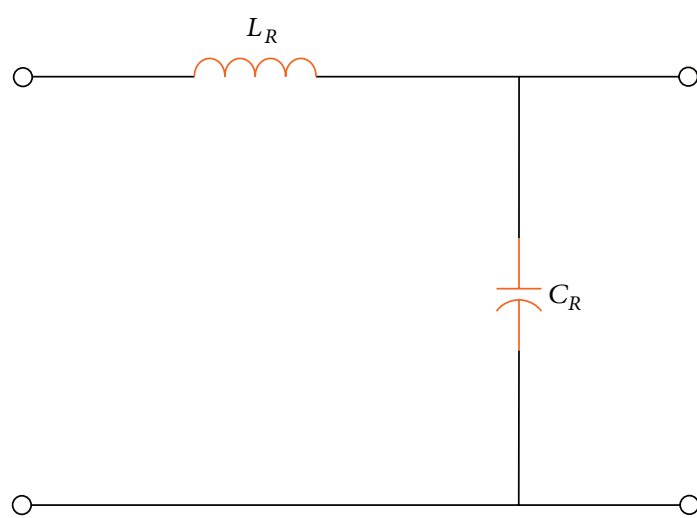

(a)

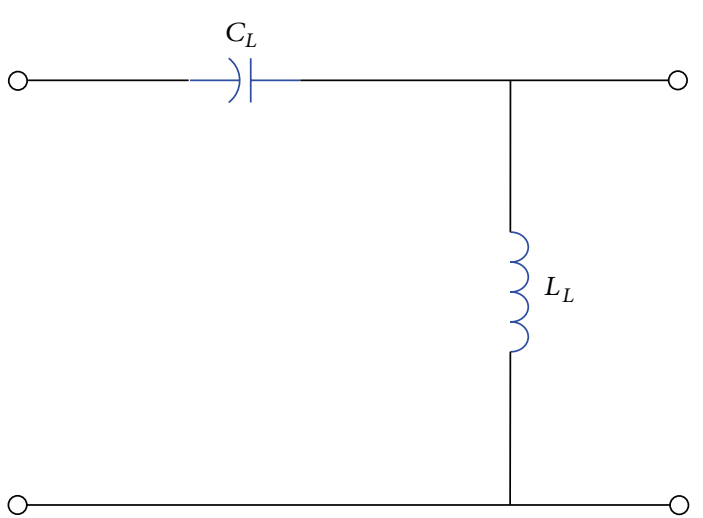

(b)

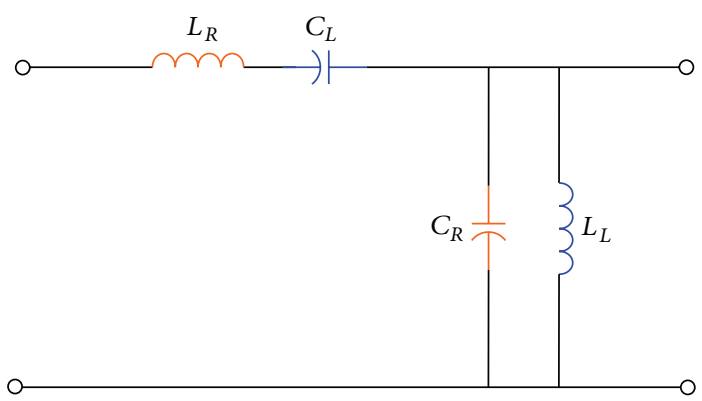

(c)

FIgURE 1: Equivalent circuits: (a) pure RH TL, (b) pure LH TL, and (c) CRLH TL.

and measurement conducted validate the proposed design principle.

\section{Theoretical Analysis}

In the ideal condition, the equivalent circuit of the pure $\mathrm{RH}$ TL and pure LH TL is depicted in Figures 1(a) and 1(b), respectively. It can be seen from Figure $1(\mathrm{a})$ that the pure $\mathrm{RH}$ TL includes series inductance $L_{R}$ and shunt capacitance $C_{R}$, which provides a low-pass filtering response. Opposite to $\mathrm{RH}$ $\mathrm{TL}$, the pure LH TL is composed of series capacitance $C_{L}$ and shunt inductance $L_{L}$ and indicates a high-pass response, as shown in Figure 1(b). The cutoff frequencies of these two responses can be expressed as

$$
\begin{aligned}
& \omega_{c R}=\frac{1}{\sqrt{L_{R} C_{R}}}, \\
& \omega_{c L}=\frac{1}{\sqrt{L_{L} C_{L}}},
\end{aligned}
$$

where subscripts $R$ and $L$ represent RH and LH, respectively.

In reality, a pure $\mathrm{LH}$ structure is not possible because of unavoidable RH parasitic parameters. Therefore, a CRLH structure represents the most general form of a structure with attributes. Figure 1(c) shows the general model of CRLH TL, which consists of inductance $L_{R}$ in series with capacitance $C_{L}$ and shunt capacitance $C_{R}$ in parallel with inductance $L_{L}$. Thus, when $w_{c L}<w_{c R}$, a bandpass characteristic can be constructed under the balanced case. The center frequency of the passband $w_{0}$ can be calculated by

$$
\omega_{0}=\sqrt{\omega_{c R} \omega_{c L}}=\frac{1}{\sqrt[4]{L_{R} C_{R} L_{L} C_{L}}} .
$$

Because the passband is formed by two parts, that is, $\mathrm{LH}$ part and $\mathrm{RH}$ part, the bandwidth can be enlarged and a wideband response is always provided. Certainly, the other EM properties can also be achieved when $w_{c L} \geq w_{c R}$.

\section{Filter Implementation}

Based on the discussion above, a wide-band BPF can be designed with CRLH structure, and a wider bandwidth can be realized with larger $L_{L}, C_{L}$ and smaller $L_{R}, C_{R}$. Therefore, a symmetrical circuit model of improved CRLH structure is proposed and illustrated in Figure 2. It can be seen that there are two shunt tanks such that a larger $L_{L}$ will be provided. Moreover, it should be noted that an additional capacitor $C_{R 1}$ is added to the branch of $L_{L}$, which avoids the grounded operation directly and realizes the all-planar structure.

Figure 3 shows the corresponding microstrip structure of circuit model in Figure 2. It can be observed that the structure is mainly made up of three parts, that is, interdigital structure, meander lines, and rectangle patch. In this work, the series tank is realized with microstrip interdigital capacitor, and the shunt tank is implemented by meander line inductor and rectangle patch capacitor. The interdigital capacitor has a relatively larger value of the series capacitor $C_{L}$, while 


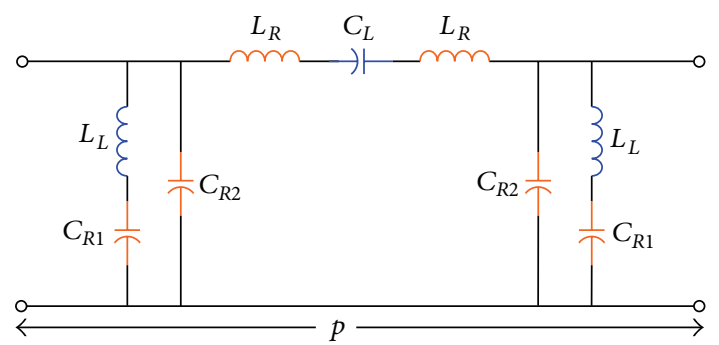

FIGURE 2: Symmetrical equivalent circuit of the improved CRLH structure.

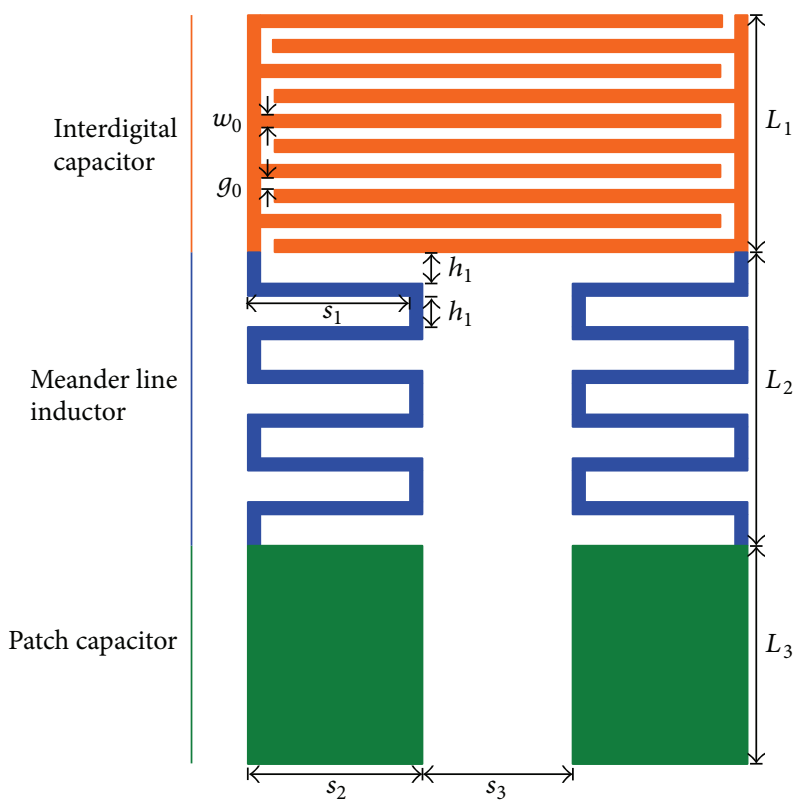

FIgURE 3: Microstrip implementation of the improved CRLH structure.

two meander lines provide a large shunt inductor $L_{L}$, which ensure the wide-band BPF condition $w_{c L}<w_{c R}$. The other parameters are carefully selected to meet the balanced CRLH requirement, which averts the bandgap in the wide passband range.

EM simulated software Sonnet is applied to simulate the designed filter and optimize its size parameters. The TaconicRF-35A2 substrate with a relative dielectric constant of 3.5 and a thickness of $0.76 \mathrm{~mm}$ is used. In this paper, a wide-band $\mathrm{BPF}$ with passband ranges from $1.9 \mathrm{GHz}$ to $3.3 \mathrm{GHz}$ (total $3 \mathrm{~dB}$ bandwidth is $1.4 \mathrm{GHz}$ ) will be designed. According to the parameters extracted and converted method described in $[16,17]$, the filter size can be obtained and finally optimized by Sonnet as follows: $w_{0}=g_{0}=0.2, s_{1}=2.6, s_{2}=2.8, s_{3}=3$, $h_{1}=0.5, h_{2}=3.5, L_{1}=3.8, L_{2}=4.7$, and $L_{3}=3.5$ (unit: millimeters).

\section{Results and Discussion}

For demonstration purpose, the well-designed wide-band filter is fabricated on Taconic-RF-35A2 substrate. Without

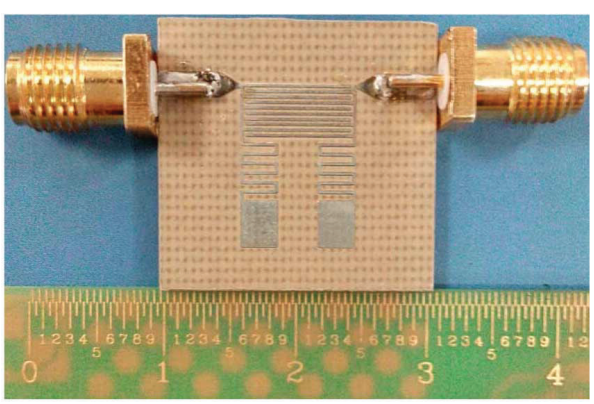

Figure 4: Photograph of the proposed filter.

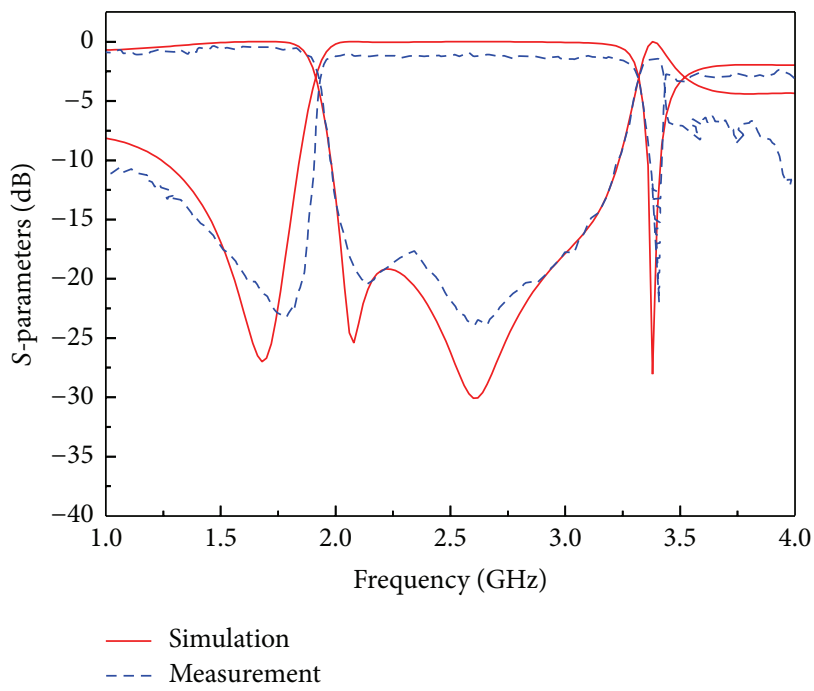

FIGURE 5: Measured and simulated responses of the wide-band filter.

the via hole, it is easy to process the circuit with ordinary technology. Figure 4 depicts the photograph of the fabricated filter. The tapped coupling structure is used to design the input/output structure and wedge shape is introduced for impedance matching. The fabricated filter is measured by network analyzer of CETC AV3629.

A comparison between simulation and measurement is presented in Figure 5, where the red solid lines and blue dashed lines indicate the simulated and measured results, respectively. A wide-band bandpass response with the passband ranges from 1.91 to $3.32 \mathrm{GHz}$ is obtained and the corresponding $3 \mathrm{~dB}$ bandwidth is about $51.9 \%$. The maximum measured insertion loss is approximately $1.38 \mathrm{~dB}$ and the return loss in passband is better than $17 \mathrm{~dB}$. Two transmission zeros located at lower and upper side of passband enhance the filter selectivity. A good agreement obtained between the measured and simulated results indicates the validity of the design principle. Some discrepancy between the measured and simulated data can be attributed to the inevitable inaccuracy in fabrication and larger dielectric loss at high frequency.

Moreover, the complex propagation constant $\gamma$ will be discussed to interpret behaviors of the proposed CRLH 


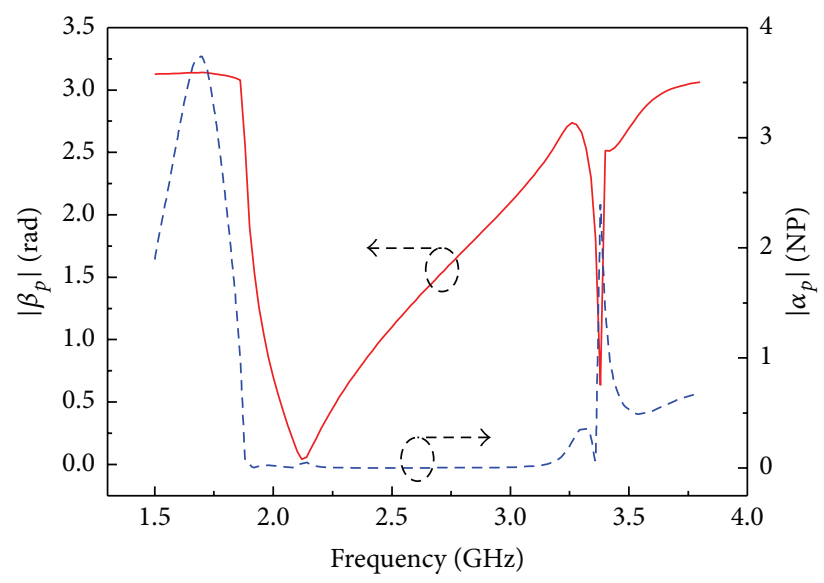

FIGURE 6: Dispersion characteristic diagram of the designed filter with improved CRLH structure.

device. According to circuit network analysis, $\gamma(w)$ can be expressed as

$$
\gamma(\omega)=\alpha(\omega)+j \beta(\omega)=\frac{\cos ^{-1}(A)}{p},
$$

where $\alpha(w)$ and $\beta(w)$ represent the attenuation factor and propagation constant, respectively. $p$ is the total length of improved CRLH filter. Parameter $A$ is a matrix element of $\mathrm{ABCD}$-matrix and it can be obtained from

$$
A=\frac{1-S_{11} S_{22}+S_{12} S_{21}}{2 S_{21}} .
$$

Therefore, $\gamma(w)$ can be easily calculated from the simulated or measured $S$ parameters.

Figure 6 shows $\gamma(w)$ of the designed wide-band filter with CRLH TL structure. In general, the bigger the attenuation factor $\alpha$ is, the greater the electromagnetic wave is attenuated. If attenuation factor $\alpha=0$, a passband will be presented since $\gamma(w)=j \beta(w)$ is an imaginary. Otherwise, a stopband occurs in certain frequency range. So, a passband occurs within the frequency ranges from 1.9 to $3.3 \mathrm{GHz}$. On the other hand, it can be found that the product of group velocity and phase velocity $v_{g} v_{p}<0\left(v_{g}=\partial \omega / \partial \beta, v_{p}=\omega / \beta\right)$ in the frequency range from 1.91 to $2.12 \mathrm{GHz}$ and shows the $\mathrm{LH}$ performance. Similarly, it can be obtained that $v_{g} v_{p}>0$ in the frequency range from 2.12 to $3.32 \mathrm{GHz}$ and indicates the $\mathrm{RH}$ performance.

\section{Conclusions}

In this paper, a wide-band BPF is designed with the improved CRLH TL. Additional patch capacitance is added to remove traditional via hole, which reduces the fabrication difficulty and circuit loss. The filter is simulated and optimized by EM software Sonnet. Good agreement between simulation and measurement validates the validity of the proposed design principle. To observe the LH property, the dispersion diagram is given and explained in the paper.

\section{Competing Interests}

The authors declare that there are no competing interests regarding the publication of this paper.

\section{Acknowledgments}

This work was supported by the National Natural Science Foundation of China under Grant 61461020 and International Cooperation Funds and Science (nos. 20133BDH80007, 20141BDH80002) and Technology Innovation Team of Jiangxi Province (no. 20142BCB24004) and Research Funds of East China Jiaotong University (no. 15XX03).

\section{References}

[1] N. Engheta and R. W. Ziolkowsiki, Electromagnetic Metamaterials Physics and Engineering Explorations, Wiley and IEEE Press, 2006.

[2] "Breakthrough of the year. The runners-up," Science, vol. 338, no. 6114, pp. 1525-1532, 2012.

[3] R. A. Shelby, D. R. Smith, and S. Schultz, "Experimental verification of a negative index of refraction," Science, vol. 292, no. 5514, pp. 77-79, 2001.

[4] A. Lai, C. Caloz, and T. Itoh, "Composite right/left-handed transmission line metamaterials," IEEE Microwave Magazine, vol. 5, no. 3, pp. 34-50, 2004.

[5] C. Caloz and T. Itoh, "Application of the transmission line theory of left-handed (LH) materials to the realization of a microstrip 'LH line"' in Proceedings of the IEEE Antennas and Propagation Society International Symposium, vol. 2, pp. 412415, IEEE, usa, June 2002.

[6] G. V. Eleftheriades, O. Siddiqui, and A. K. Iyer, "Transmission line models for negative refractive index media and associated implementations without excess resonators," IEEE Microwave and Wireless Components Letters, vol. 13, no. 2, pp. 51-53, 2003.

[7] C. Caloz and T. Itoh, Electromagetic Metamaterials: Transmission Line Theory and Microwave Applications, John Wiley \& Sons and IEEE Press, 2006.

[8] W. J. Feng, W. Q. Che, and Q. Xue, "Transversal signal interaction: overview of high-performance wideband bandpass filters," IEEE Microwave Magazine, vol. 15, no. 2, pp. 84-96, 2014.

[9] H. Wang, Q.-X. Chu, and J.-Q. Gong, "A compact wideband microstrip filter using folded multiple-mode resonator," IEEE Microwave and Wireless Components Letters, vol. 19, no. 5, pp. 287-289, 2009.

[10] S.-J. Sun, B. Wu, T. Su, K. Deng, and C.-H. Liang, "Wideband dual-mode microstrip filter using short-ended resonator with centrally loaded inductive stub," IEEE Transactions on Microwave Theory and Techniques, vol. 60, no. 12, pp. 36673673, 2012.

[11] S. Sun, L. Zhu, and H.-H. Tan, "A compact wide-band bandpass filter using transversal resonator and asymmetrical interdigital coupled lines," IEEE Microwave and Wireless Components Letters, vol. 18, no. 3, pp. 173-175, 2008.

[12] R. Gómez-García, M. Sánchez-Renedo, B. Jarry, J. Lintignat, and B. Barelaud, "A class of microwave transversal signalinterference dual passband planar filters," IEEE Microwave and Wireless Components Letters, vol. 19, no. 3, pp. 158-160, 2009.

[13] B. Li, X. W. Dai, B. Wu, and C. H. Liang, "Ultra wideband filter design based on composite right-/left-handed transmission 
line," Microwave and Optical Technology Letters, vol. 49, no. 10, pp. 2379-2381, 2007.

[14] S.-H. Oh, K.-T. Kim, J.-H. Lee, S. Kahng, and H.-S. Kim, “Design of the miniaturized ultra-wide band (UWB) filter using the metamaterial characteristic," in Proceedings of the 3rd AsiaPacific Microwave Conference (APMC '13), pp. 954-956, IEEE, November 2013.

[15] H. W. Liu, P. Wen, S. S. Zhu, B. P. Ren, and Y. S. He, "Hightemperature superconducting composite right/left-handed resonator," IEEE Trasaction on Applied Superconductivity, vol. 26, no. 3, Article ID 1500204, 2016.

[16] B. Li, J. P. Yang, and W. Wu, "A planar microstrip implementation of dual-composite right/left handed transmission line," in Proceedings of the International Conference on Microwave and Millimeter Wave Technology (ICMMT'08), pp. 1617-1619, IEEE, Nanjing, China, April 2008.

[17] J. S. Hong and M. J. Lanaster, Microwave Filter for RF/Microwave Application, John Wiley \& Sons, New York, NY, USA, 2001. 


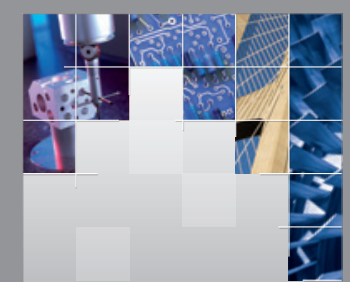

\section{Enfincering}
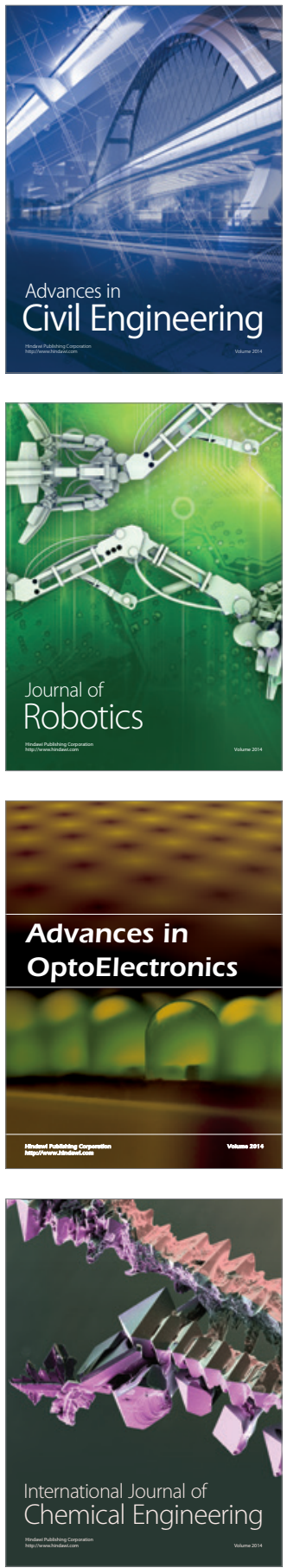

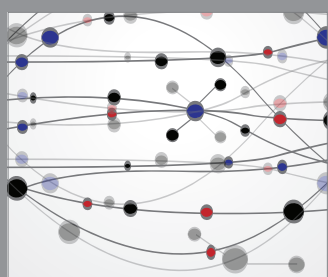

The Scientific World Journal

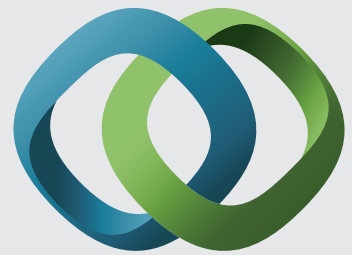

\section{Hindawi}

Submit your manuscripts at

http://www.hindawi.com
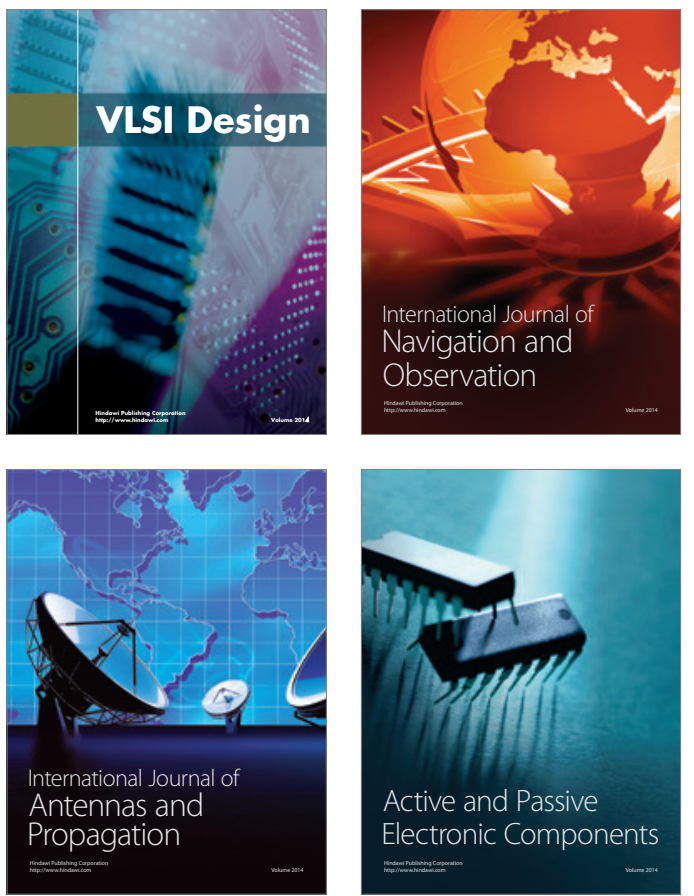
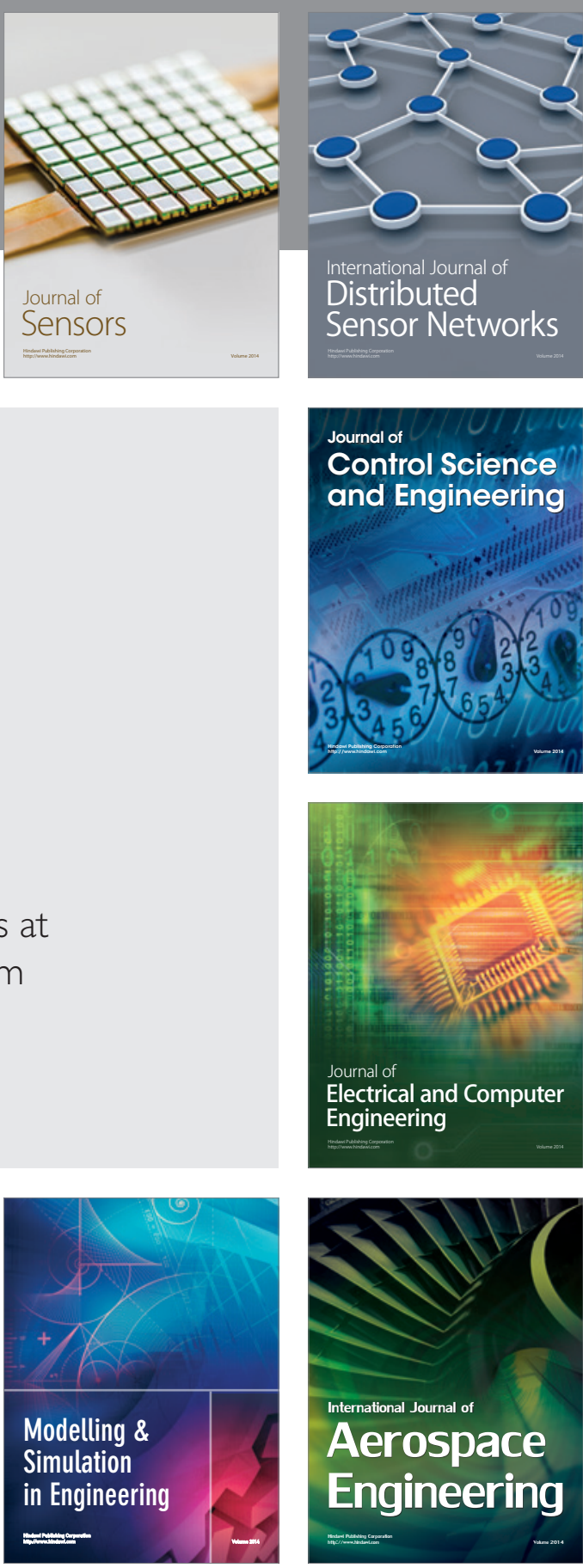

International Journal of

Distributed

Sensor Networks

Journal of

Control Science

and Engineering
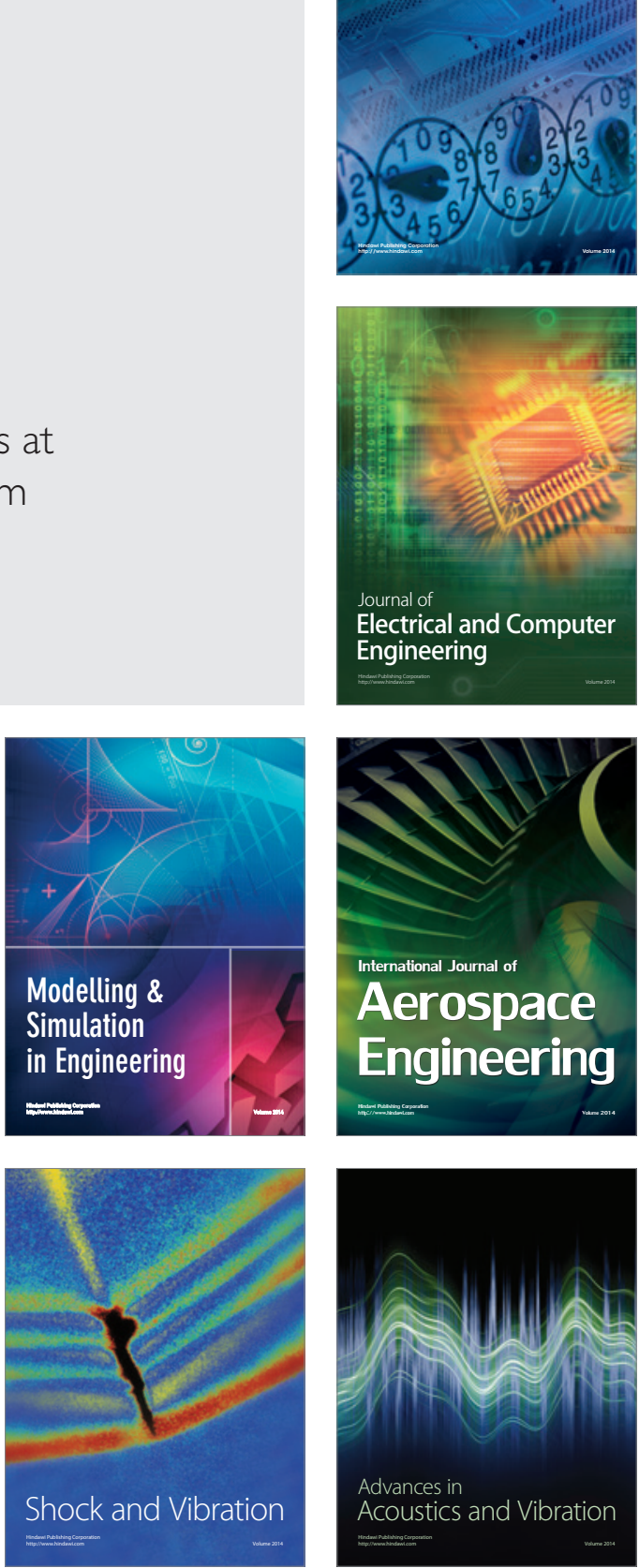